\title{
Low-Frequency Beam-Plasma Interactions in a Finite-Sized Plasma
}

\author{
V. P. Bhatnagar and W. D. Getty \\ Electron Physics Laboratory, Department of Electrical and Computer Engineering, The University of Michigan, \\ Ann Arbor, Michigan 48104
}

(Received 1 December 1971; final manuscript received 8 June 1972)

\begin{abstract}
Results are presented for an experimental and theoretical investigation of low-frequency waves in a beam-generated plasma. Waves are excited at frequencies immediately above the ion-plasma frequency by sinusoidally modulating the electron beam current. The study is aimed at obtaining ion heating at the lower-hybrid resonant frequency. An electron beam of 400-1000 V energy and a few milliamperes of current is used to generate plasmas with densities in the range of $10^{9} \mathrm{~cm}^{-3}$ in a magnetic field of a few hundred gauss. Hydrogen, argon, deuterium, and neon gases are used. The rf electric field is probed as a function of $r, z$, and frequency, and energetic ions are detected by a gridded probe. Two or three resonances are found in the probe response above $\omega_{p i}$, and are shown to be multiple half-wavelength resonances of standing plasma waveguide axisymmetric modes. A normal-mode analysis is used to calculate the probe responses and is found to correctly predict the effects of varying beam voltage, plasma density, ion mass, and dc axial magnetic field. The excited wave may be viewed as an electrostatic wave propagating at an angle very close to $90 \mathrm{deg}$ from the magnetic field.
\end{abstract}

\section{INTRODUCTION}

The investigation reported in this paper is concerned with the generation of a large low-frequency radial if electric field in a plasma for the purpose of ion heating. The main objective of the work is to understand the basic mechanism of excitation of the rf electric field and its propagation characteristics. Only small-signal behavior is studied and no efforts are directed toward achieving a very energetic plasma. Experimental and theoretical evidence will be presented which shows that a modulated electron beam excites resonant modes of a beam-generated plasma, and that at the resonant frequencies a relatively large radial rf electric field is excited in the plasma which produces ion heating. The excitation frequency is near the electron-ion lowerhybrid frequency $\left(\omega_{1 \mathrm{~h}}\right)$.

At low frequencies the electron beam-plasma instability growth rates are small and relatively weak interactions are obtained. Because of small instability growth rates one does not generally obtain ion heating simply by injecting an unmodulated electron beam into a plasma."The point of view taken by Haas and Eisner ${ }^{1,2}$ and the present authors ${ }^{3,4}$ is that a modulated electron beam creates an rf charge perturbation in the plasma which drives a large radial electric field at the modulation frequency. The frequency is chosen to give the most effective coupling to the plasma ions, e.g., the ion-cyclotron, ion-ion hybrid, or ion-electron hybrid resonant frequencies. A rapidly growing two-stream instability at the chosen frequency is thus not required to achieve a coupling between the electron beam and plasma ions.

Other experimental studies of waves near $\omega_{\mathrm{lh}}$ have been carried out in beam-plasma systems. Haas and his colleagues have reported several experiments concerning ion heating in a beam-generated plasma using a modulated electron beam. ${ }^{1,2,5-7}$ They observed that ion heating is maximum at $\omega \cong \omega_{\mathrm{lb}}$ in a beam-generated plasma in mirror geometry. Ion coupling was also observed when the beam was modulated at $\omega_{c i}$ in their experiments. Observations of beam-driven ion oscillations at the ion-ion hybrid frequency for atomic and molecular hydrogen have also been reported. ${ }^{8}$ The production of energetic ions is caused by the acceleration of individual ions in the rf field. ${ }^{1,3}$

The effect of finite wavelength along the magnetic axis is important in the theory and experiment to be described in the present paper. In a cold plasma, the resonant frequencies of a plane wave that propagates at an arbitrary angle $\theta$ with respect to the magnetic axis are given by the solutions of the bicubic equation ${ }^{9}$ :

$$
\begin{array}{r}
{\left[\left(1+\cot ^{2} \theta\right) \omega^{2}-\left(\omega_{p e}{ }^{2}+\omega_{p i}{ }^{2}\right) \cot ^{2} \theta\right]\left(\omega^{2}-\omega_{c i}{ }^{2}\right)\left(\omega^{2}-\omega_{c s}{ }^{2}\right)} \\
-\omega^{2}\left(\omega_{p e}{ }^{2}+\omega_{p i}{ }^{2}\right)\left(\omega^{2}-\omega_{c e} \omega_{c i}\right)=0 .
\end{array}
$$

The solution for $\omega^{2}$ from (1) which is of interest for the present discussion is approximately given by

$$
\omega_{\mathrm{lh}}{ }^{2}(\theta)=\omega_{p i}{ }^{2} \frac{1+(M / m) \cot ^{2} \theta}{1+\cot ^{2} \theta},
$$

when $\omega_{c i}{ }^{2} \gg \omega_{p e}{ }^{2} \gg \omega_{c e} \omega_{c i}$. This reduces to $\omega_{1 \mathrm{l}}=\omega_{p i}$ when $\theta=90 \mathrm{deg}$, and becomes independent of ion mass when $\cot \theta \gg(m / M)^{1 / 2}$. At high densities, (1) gives

$$
\omega_{\mathrm{lh}}{ }^{2}(\theta) \cong \omega_{c e} \omega_{c i}\left[1+(M / m) \cot ^{2} \theta\right],
$$

which also tends to rise rapidly and becomes independent of ion massas $\theta$ changes from $90 \mathrm{deg}$. The value of $\theta$ is determined by the geometry of the plasma and the boundary conditions, and to evaluate it accurately the field equations and boundary-value problem must be solved. It has previously been pointed out that within the quasistatic approximation $(\nabla \times E \cong 0)$ the various branches of the guided-wave dispersion curves to be given in Sec. III can be considered to be plots of the resonance condition for plane waves as given by (1) in the $\omega-k_{z}$ plane with $\theta$ as the parameter along each branch., ${ }^{40}$ In the present work it is found that the 


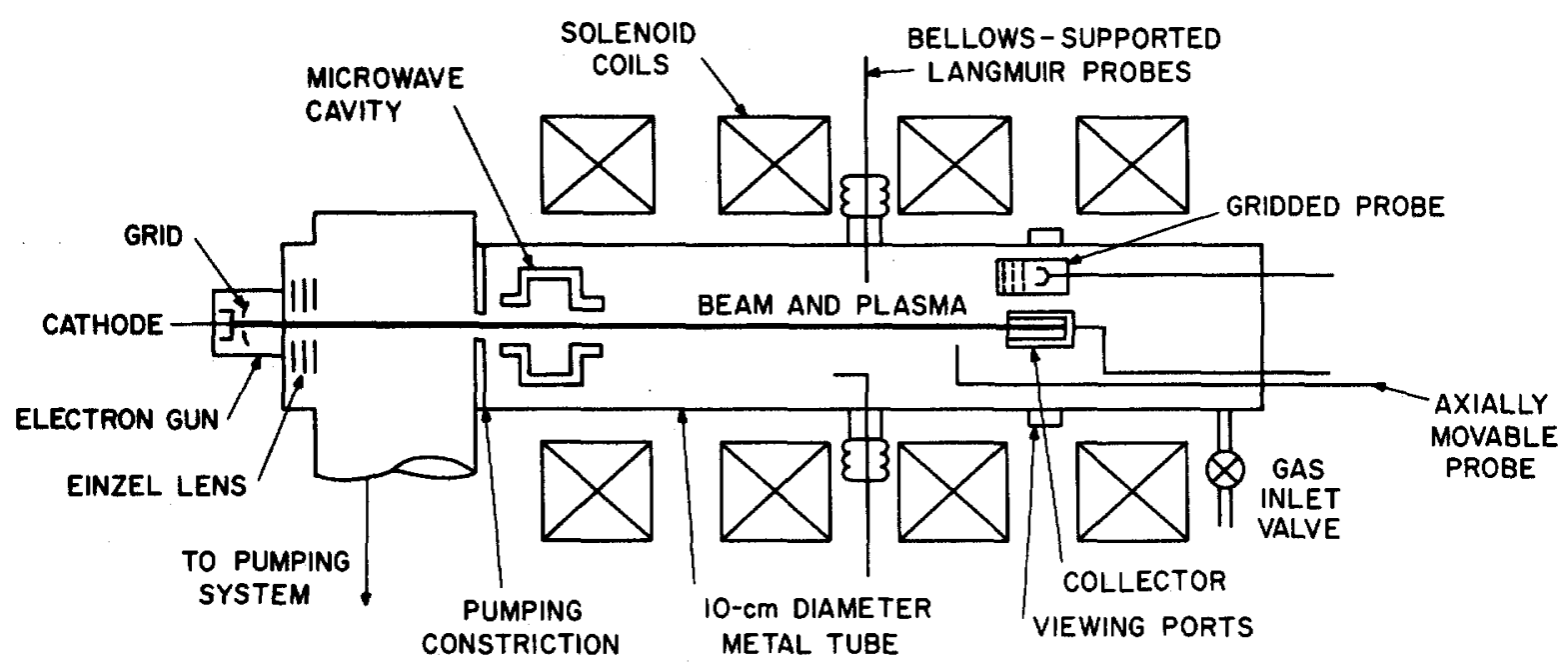

FrG. 1. Schematic diagram of the experimental apparatus.

waves excited by the electron beam may occur at frequencies where $\theta$ satisfies the condition $\cot \theta \gg(m / M)^{1 / 2}$ in (2) and, therefore, the wave properties are nearly independent of ion mass. ${ }^{4}$

\section{EXPERIMENT}

A schematic diagram of the experimental setup is shown in Fig. 1. The vacuum system consists of a differentially pumped central vacuum chamber for the plasma and a low-pressure region for the electron gun. The plasma chamber is a $10-\mathrm{cm}$ diam cylindrical stainless steel tube approximately $1 \mathrm{~m}$ in length. For plasma generation, hydrogen, deuterium, neon, or argon is continuously admitted into the plasma chamber through a needle valve. The electron gun is a Pierce traveling-

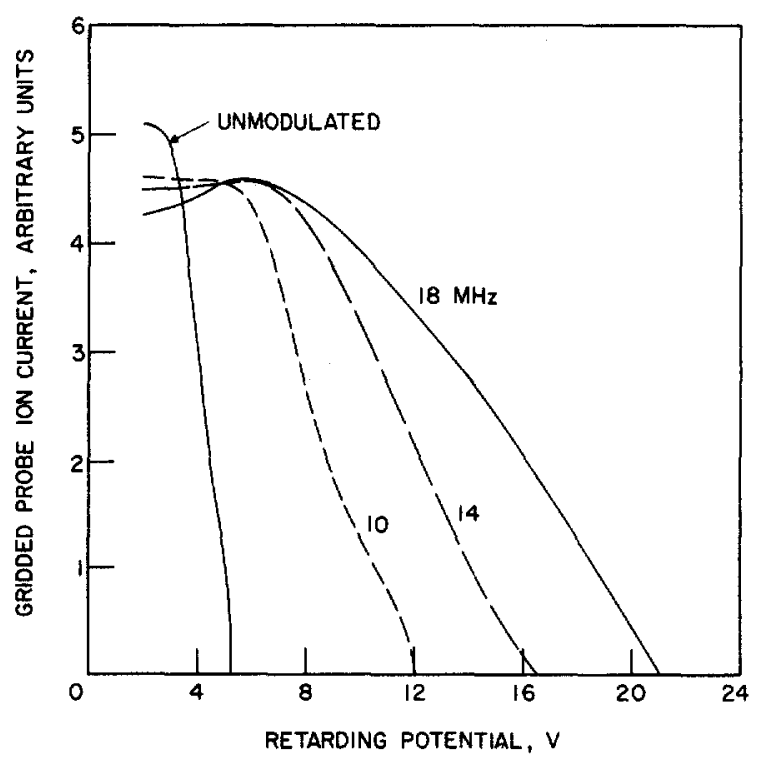

FIG. 2. Gridded probe retarding potential curves with different beam-current modulation frequencies. wave tube gun with a grid located very near the cathode. Any $\mathrm{cw}$ or modulated rf signal can be applied at the grid electrode for current modulation. The upper frequency limit is set by cathode lead inductance and is approximately $30 \mathrm{MHz}$. The relatively weak electron beam generates a "quiescent" low-density plasma in the gas-filled chamber. ${ }^{11}$ Visual observation indicates that the beam and plasma are approximately $6 \mathrm{~mm}$ in diameter. Typical parameters of the beam and plasma are beam voltage, $400-1000 \mathrm{~V}$; beam current, 1.5-2.5 $\mathrm{mA}$; gas pressure, $8 \times 10^{-5}-8 \times 10^{-4}$ Torr; plasma density, $5 \times 10^{8}-5 \times 10^{9} / \mathrm{cm}^{3}$; electron temperature, $5-6 \mathrm{eV}$; and axial magnetic field, $300-400 \mathrm{G}$. The electron beam is collected by a metal collector, and the rf current modulation on the beam is monitored on a highfrequency oscilloscope by returning the collector beam current to ground through a $50-\Omega$ resistor.

Plasma density is measured with a Langmuir probe and a microwave cavity in the $\mathrm{TM}_{010}$ mode. The densities measured by the two methods were compared and good agreement was found. Two coaxially shielded wire probes are used for rf field detection. One of the $\mathrm{rf}$ probes is radially movable and the other is axially movable. The rf probe signal is fed to a matched preamplifier with $40 \mathrm{~dB}$ of voltage gain and detected with an $\mathrm{rf}$ millivoltmeter. The axially movable $\mathrm{rf}$ probe is used for interferometric measurements of the axial wavelength. A double balanced if mixer whose dc output is a function of the phase difference between the reference and the detected rf probe signal is used to measure the axial wavelength. Both rf probes are used to measure the electric field as a function of frequency.

A retarding field electrostatic analyzer (gridded probe) is used to detect the presence of energetic charged particles. The gridded probe points in the axial direction and is movable axially. The size of the gridded probe is comparable to the size of the plasma and the probe was located off-axis so that it did not 


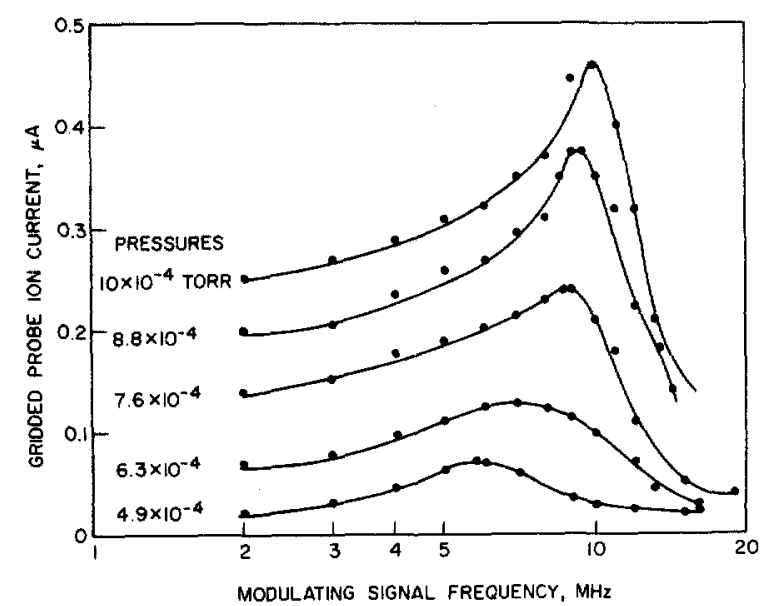

FIG. 3. Gridded probe collector current as a function of beammodulation frequency for various pressures. $\left(V_{b}=500 \mathrm{~V}, I_{b}=\right.$ $2 \mathrm{~mA}, B_{0}=275 \mathrm{G}$, mirror ratio $=2.5: 1$.)

disturb the plasma. Because of the off-axis location the probe intercepted mostly energetic ions. Consequently, it could not be used for ion temperature measurements, but it was useful for detecting the presence of energetic ions at various frequencies or retarding voltages. In order to determine the frequency response of the system to the current-modulated electron beam, a constant-amplitude sinusoidal current modulation was carefully maintained over the desired frequency band ( 2 to $25 \mathrm{MHz}$ ) by adjusting the rf grid voltage according to a calibration curve that compensated for the gun cathode lead inductance. The calibration curve gave the if grid voltage required at each frequency for constant rf beam collector current in the absence of a plasma. The calibration curve thus obtained was used when measuring the rf probe current and the griddedprobe ion current as functions of frequency in the presence of a plasma.

The rf current modulation has an appreciable effect on the gridded probe retarding potential curve. Figure 2 shows the retarding potential curves for the unmodulated case and for modulation frequencies of 10, 14, and $18 \mathrm{MHz}$. The energy spread increases considerably when the frequency of modulation is $18 \mathrm{MHz}$. The spread in ion energies is over $20 \mathrm{~V}$ for the particular case shown here. Similar results are found under all operating conditions but the frequency at which the maximum effect occurs varies with operating conditions. The increase in the ion-energy spread indicated by the gridded probe is also accompanied by an increase in the visual diameter of the plasma. Thus, it is concluded that energetic ions are produced when the beam is modulated at certain frequencies.

The gridded probe response as a function of frequency is shown by the curve of Fig. 3. The curves shown are for a retarding potential of $5 \mathrm{~V}$. As the pressure is increased the frequency of the peak in these curves moves toward higher frequencies. The variation of the peak frequency, or "resonant" frequency, with pressure is interpreted as a variation with plasma density. The plasma density was measured with the Langmuir probe and the ion plasma frequency was calculated. The results show that the resonant frequency is always above the calculated ion plasma frequency. Any error in the Langmuir probe density measurement and, therefore, the calculated ion plasma frequency is expected to overestimate the density in the method used to interpret the Langmuir probe curves. ${ }^{12}$

The radially movable of probe was located at a fixed axial position and was used to detect the radial rf electric field as a function of frequency. The probe tip was located approximately one plasma diameter away from the plasma axis. Effects of variations of plasma density, beam voltage, ion mass, and magnetic field were studied. A typical probe response is shown in Fig. 4 in which the amplitude of the radial rf electric field is plotted as a function of beam-current modulation frequency. The frequency response of the probe to the modulated electron beam in high vacuum (i.e., no plasma) is also shown. In the presence of plasma, two or three broad peaks or resonances are typically observed in the probe response at frequencies which are, in general, not harmonically related. The third peak was usually observed but was not as prominent as the first and second peaks. A set of these curves was taken as the plasma density was varied by changing the gas pressure. Other parameters such as beam voltage, beam current, and magnetic field were held constant. As the density is increased the resonant frequencies shift upward. The plasma density measured by the Langmuir probe indicates that the first resonant frequency is just above the ion-plasma frequency.

Frequency response curves for different beam voltages were taken at constant beam current and plasma density. It is found that the first two resonant frequencies decrease as the beam voltage is increased. The change in the first resonant frequency is smaller than the change in the second resonant frequency for the same change in the beam voltage.

Frequency response curves similar to those for hydrogen were obtained for deuterium, neon, and argon gases. It is found that the resonant frequencies increase with an increase in plasma density. By comparing the resonant frequencies obtained in hydrogen, neon, and argon plasmas for the same plasma density, it is found that the resonant frequencies for neon and argon plasmas are slightly smaller than the corresponding resonant frequencies for the hydrogen plasma. However, the changes in the resonant frequencies are much smaller than the changes in the ion-plasma frequencies in the two cases. Thus, it is concluded that the resonant frequencies are relatively independent of the ion mass. It was also found that the resonant frequencies do not change significantly with the dc magnetic field.

The if electric field outside the plasma was found to fall monotonically as a function of radius. The reso- 
Frg. 4. Experimentally observed radial electric-field amplitude as a function of frequency for different densities. $\left(V_{b}=600 \mathrm{~V}, I_{b}=\right.$ $2 \mathrm{~mA}, P_{0}=335 \mathrm{G}$, hydrogen gas.)

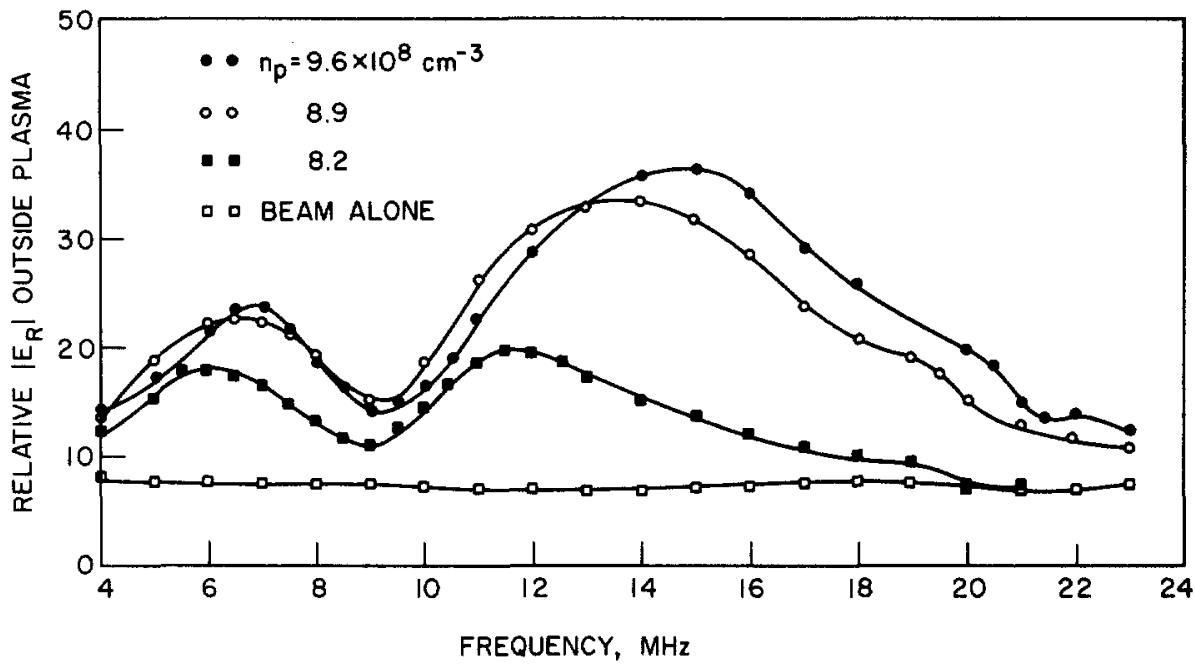

nance effect in frequency response is also observed in these data. The rf probes werc also used to monitor signals that might be spontaneously excited by the unmodulated electron beam. No signals of this type were found at either low frequencies near $\omega_{p i}$ or high frequencies near $\omega_{p e}$. The electron beam was too weak to produce strong beam-plasma convective or absolute instabilities that could cause spontaneous signals.

A typical measurement of the radial if electric field as a function of axial position is shown in Fig. 5 at the first and second resonant frequencies. Since the probe could be moved a distance of only $10 \mathrm{in}$. from the collector, the data points near the gun end could not be taken. However, the data were sufficient to indicate that the first and second resonances are half-wave and full-wave resonances. The wavelengths thus found are much larger than the wavelength $v_{0} / f_{c e}$ corresponding to the beam-cyclotron waves. The radially and axially movable probes were positioned diametrically opposite just outside the plasma column. The if signals from the two probes were amplified by two identical voltage amplifiers and fed to a double-beam oscilloscope. Comparison of the phases showed that the rf fields are in phase, suggesting that the mode is axisymmetric.

\section{BEAM-PLASMA SYSTEM MODEL}

The dispersion relation studied in this section is for a radially bounded but axially infinite beam-plasma system. The frequency range studied is near the lowerhybrid frequency. The roots of the dispersion equation are used in carrying out normal-mode field calculations for an axially bounded system. The method used is similar to that used by Frey and Birdsall for the determination of complex eigenfrequencies in a bounded
Fic. 5. Real part of the radial electric field at $r=2 b$ as a function of axial distance for experimental and theoretical results. $\left(V_{b}=600 \mathrm{~V}, I_{b}=2.5 \mathrm{~mA}, B_{0}=\right.$ $310 \mathrm{G}, n_{p}=1 \times 10^{\circ} / \mathrm{cm}^{2}, L=61 \mathrm{~cm}, b=$ $3 \mathrm{~mm}, \nu_{e N} / \omega_{p i}=0.1$, hydrogen gas.)

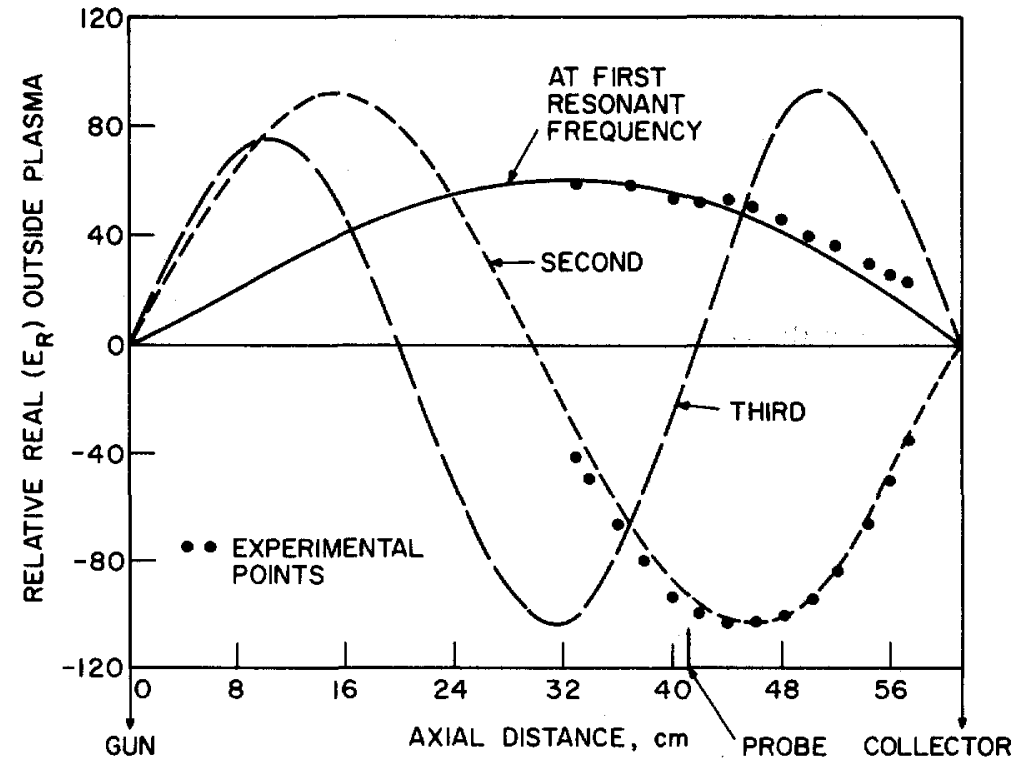




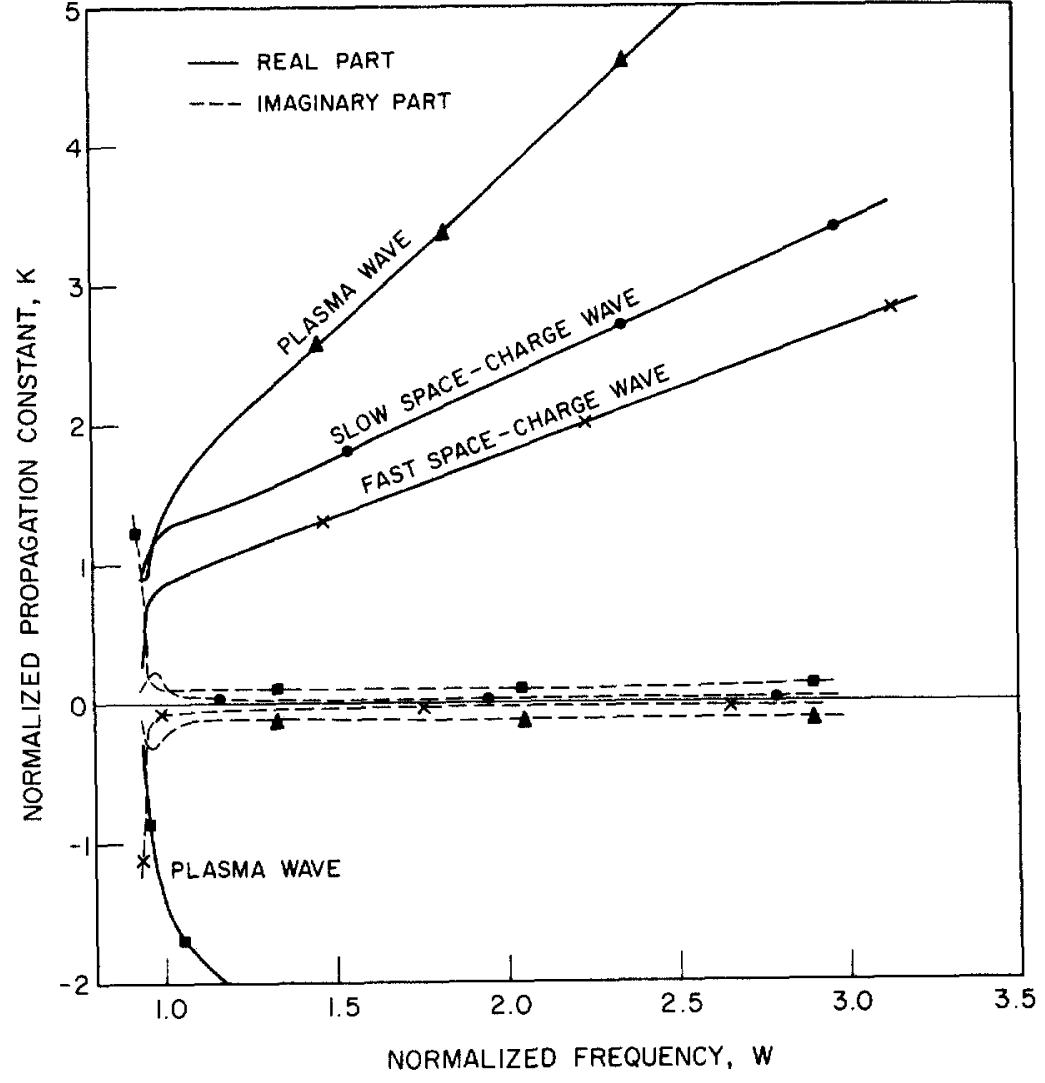

Fic. 6. Propagation constant as a function of frequency for an open beam-plasma waveguide for the parameters of the experiment. The imaginary parts of the two spacecharge wave roots are exaggerated for $W>1.2$ to make them visible on the diagram. $\left(V_{b}=500 \mathrm{~V}, \omega_{c e} / \omega_{p e}=10\right.$, $\nu_{e N} / \omega_{p i}=0.1, \omega_{p b}^{2} / \omega_{p_{i}}^{2}=500$.) beam-plasma system. ${ }^{13}$ The cold plasma model includes ion motion, dc magnetic field $\mathbf{B}_{0}$ along the $z$ axis, electron-neutral collisions, and finite transverse dimensions. The axial phase velocities of the waves which are involved are all much higher than thermal velocities, $\omega \gg k_{z} v_{T}$; therefore, temperature effects are neglected.

The dispersion equation used is that of the "open" beam-plasma waveguide consisting of a beam and plasma column of equal diameter surrounded by an infinite isotropic dielectric or a vacuum. Experimentally, this situation exists when the plasma is generated by the beam itself in a relatively large-diameter waveguide. The open beam-plasma waveguide is a reasonable approximation for the present experiment, where the metal vacuum tube diameter is 17 times the plasma diameter.

First-order perturbations are taken to vary as $\exp \left[j\left(\omega t-m \phi-k_{z} z\right)\right]$, where $\phi$ is the azimuthal angle coordinate and $m=0, \pm 1, \pm 2, \cdots$. Under the quasistatic approximation, the electric field vector can be written as the gradient of a scalar potential $\Phi$ :

$$
\mathbf{E}=-\boldsymbol{\nabla} \Phi,
$$

which with Gauss's law leads to a modified Laplace's equation:

$$
\nabla \cdot K \cdot \nabla \Phi=0 .
$$

The boundary conditions used at the beam-plasma interface with the surrounding vacuum at $r=b$ are the continuity of $\Phi$ and the normal component of the displacement vector $\mathrm{D}=\mathrm{K} \cdot \epsilon_{0} \mathrm{E}$. The dispersion equation resulting from the boundary conditions is given for the $m=0$ mode by ${ }^{14}$

$$
\begin{aligned}
\pm j\left(K_{11}{ }^{0} K_{\perp^{0}}\right)^{1 / 2}\left[J_{1}\left(T_{1} b\right) / J_{0}\left(T_{1} b\right)\right] & \\
& =K_{\mathbf{1}}\left( \pm k_{z} b\right) / K_{0}\left( \pm k_{z} b\right) .
\end{aligned}
$$

The negative and positive signs on the left-hand side give positive $\operatorname{Re}\left(k_{z}\right)$ and negative $\operatorname{Re}\left(k_{z}\right)$, respectively, for $\omega_{\mathrm{lh}}<\omega<\omega_{\text {pe }}$. The positive and negative signs in the arguments of $K_{1}$ and $K_{0}$ are to be taken accordingly as $\operatorname{Re}\left(k_{z}\right) \gtrless 0$ to keep the potential and field finite for large values of $r{ }^{15}$ The transverse propagation constant is $T_{1}$ inside the plasma and is given by

$$
T_{1}{ }^{2}=-k_{z}^{2}\left(K_{\|}{ }^{0} / K_{\perp}{ }^{0}\right),
$$

where $K_{\|}{ }^{0}$ and $K_{\perp}{ }^{0}$ are the cold plasma dielectric tensor components modified by the presence of the beam. ${ }^{14}$ Inside the beam-plasma region the potential is proportional to $J_{0}\left(T_{1} r\right)$ and outside, it is proportional to $K_{0}\left(k_{z} r\right)$.

The complete dispersion relation is given by (4), 
(5), and the equations for $K_{\perp}, K_{\mid l}, K_{\perp}{ }^{0}$, and $K_{11^{0}}$. Because of the transcendental nature of the Bessel functions, there may be an infinite number of radial modes for each azimuthal mode. For a given radial mode there are six $k_{z}$ roots for real $\omega$. A ForTRAN computer program ${ }^{16}$ was used to trace the $k_{z}$ roots (which may be complex) for real $\omega$. The computer program requires an initial guess for a root on a particular branch. Given a starting point, the computer program checks the accuracy of this point and corrects any error to a desired accuracy. It then traces the entire branch over a specified frequency range. Normalized complex propagation constant $K=k_{z} v_{0} / \omega_{p i}$ is plotted vs normalized real frequency $W=\omega / \omega_{p i}$ in a dispersion diagram for the frequency range $\omega>\omega_{\mathrm{lh}}$ as shown in Fig. 6. This diagram is for the lowest-order radial mode and does not show two roots for which $\left|k_{z}\right| \cong \omega_{c e} / v_{0}$. Small imaginary parts are present because of collisions. Experimental evidence indicated that the modes were axisymmetric and that the axial wavelength was large compared with the cyclotron wavelength $v_{0} / f_{c e}$. In addition to using only the lowestorder radial axisymmetric mode, the two roots for $\left|k_{z}\right| \cong \omega_{c e} / v_{0}$ which are the beam-cyclotron waves are also neglected and only the four roots corresponding to the beam and plasma space-charge waves are used. Higher-order radial modes could be included, in principle, but at a great cost in complexity. Their use would require additional information about the field structure over a cross section of the beam.

The aim of the normal-mode analysis is to calculate the frequency response of the beam-plasma system to the beam-current modulation. Each of the four waves retained from the solution of the dispersion equation contributes to the total electric field which is observed experimentally. Therefore, to compare the theoretically calculated and experimentally observed fields, the contribution of each wave must be taken into account. The relative amplitude of each wave which is excited is determined by applying the boundary conditions at the gun and collector end. The total field is then obtained by the superposition of the four waves in relative amounts proportional to their complex amplitudes.

The experimental system is axially bounded by the grid of the electron gun and the beam collector. The model is thus assumed to be axially bounded by perfectly conducting electrical short circuits. A constant current modulation of the beam is imposed at the gun end as was done experimentally. The experiment was conducted with low-percentage modulation to obtain sinusoidal drive; therefore, the small-signal approximation is assumed to be valid. The potential and field expressions are obtained in terms of the beam currentdensity modulation at the entrance plane $z=0$. Beam current and velocity perturbations are found from the force and continuity equations and are derived by Briggs. ${ }^{14}$ The summations over the four pertinent normal modes for the desired quantities in region $\mathrm{I}(0 \leqq$

$$
\begin{aligned}
& r \leqq b) \text { are } \\
& J_{1 b z}=J_{1 b 0} \sum_{i=1}^{4} X_{i} J_{0}\left(T_{1 i} r\right) \exp \left(-j k_{z i} z\right) \\
& v_{1 b z}=-\frac{e}{m} \frac{J_{1 b 0}}{\omega \epsilon_{0} \omega_{p b}^{2}} \sum_{i=1}^{4} X_{i}\left(\omega-k_{z i} v_{0}\right) J_{0}\left(T_{1 i} r\right) \exp \left(-j k_{z i} z\right), \\
& \Phi^{I}=\frac{J_{1 b 0}}{\omega \epsilon_{0} \omega_{p b}^{2}} \sum_{i=1}^{4} \frac{X_{i}\left(\omega-k_{z i} v_{0}\right)^{2}}{k_{z i}\left(1+\Delta_{i}\right)} J_{0}\left(T_{1 i} r\right) \exp \left(-j k_{z i} z\right) \\
& E_{r}^{I}=\frac{J_{1 b 0}}{\omega \epsilon_{0} \omega_{p b}{ }^{2}} \sum_{i=1}^{4} \frac{X_{i}\left(\omega-k_{z i} v_{0}\right)^{2}}{\left(1+\Delta_{i}\right)} \frac{T_{1 i}}{k_{z i}} J_{1}\left(T_{1 i} r\right) \exp \left(-j k_{z i} z\right),
\end{aligned}
$$

and

$$
E_{z}{ }^{I}=\frac{j J_{160}}{\omega \epsilon_{0} \omega_{p b}{ }^{2}} \sum_{i=1}^{4} X_{i}\left(\omega-k_{z i} v_{0}\right)^{2} J_{0}\left(T_{1 i} r\right) \exp \left(-j k_{z i} z\right)
$$

In the preceding expressions $J_{1 b 0}$ is an experimentally identifiable quantity and is the beam-current modulation at the grid of the gun $(r=0, z=0), X_{i}$ is the normalized amplitude of the $i$ th normal mode, and $\Delta_{i}$ is given by

$$
\Delta_{i}=\omega_{D i}{ }^{2} T_{1 i}{ }^{2} v_{0} /\left(\omega_{D i}{ }^{2}-\omega_{c e}{ }^{2}\right) k_{z i} \omega,
$$

where $\omega_{D i}=\omega-k_{z i} v_{0}$.

The boundary conditions at the two ends of the system are now imposed to obtain the coefficients $X_{i}$ which determine the relative amplitude of each excited wave. The axial boundary conditions at the axis of the beam $(r=0)$ at $z=0$ are $J_{1 b z}=J_{1 b 0,} v_{1 b z}=0$, and $\Phi^{I}=0$; at $z=L$ they are $\Phi^{I}=0$. Substitution of these boundary conditions into (6) through (8) gives a set of four linear, nonhomogeneous, simultaneous equations for the $X_{i}$ which is solved on the computer. The values of $X_{i}$ are substituted back into (6) through (10) to determine the physical quantities given by these equations. The electrostatic potential and the electric field for region II $(r \geqq b)$ outside the beam can similarly be expressed as a normal-mode summation. The complete expressions may be found in Ref. 17.

In the numerical computations the dispersion equation is first solved to obtain $k_{z i}(i=1, \cdots, 4)$ at a given $\omega$. The $k_{z i}$ are then used when the $X_{i}$ are solved for, and finally the total field and other quantities are calculated. The normalized radial and axial electricfield amplitudes in region II are plotted as functions of frequency in Fig. 7, and theoretical field distributions are plotted as functions of $z$ in Fig. 5. Similar curves are found for the field in region $\mathrm{I}$. Three peaks in the frequency response curves of Fig. 7 are identified. The third peak in the radial electric-field response is not as prominent as the first and second peaks. Similarly, the first peak in the axial electric-field response is not pronounced but the third peak has a 


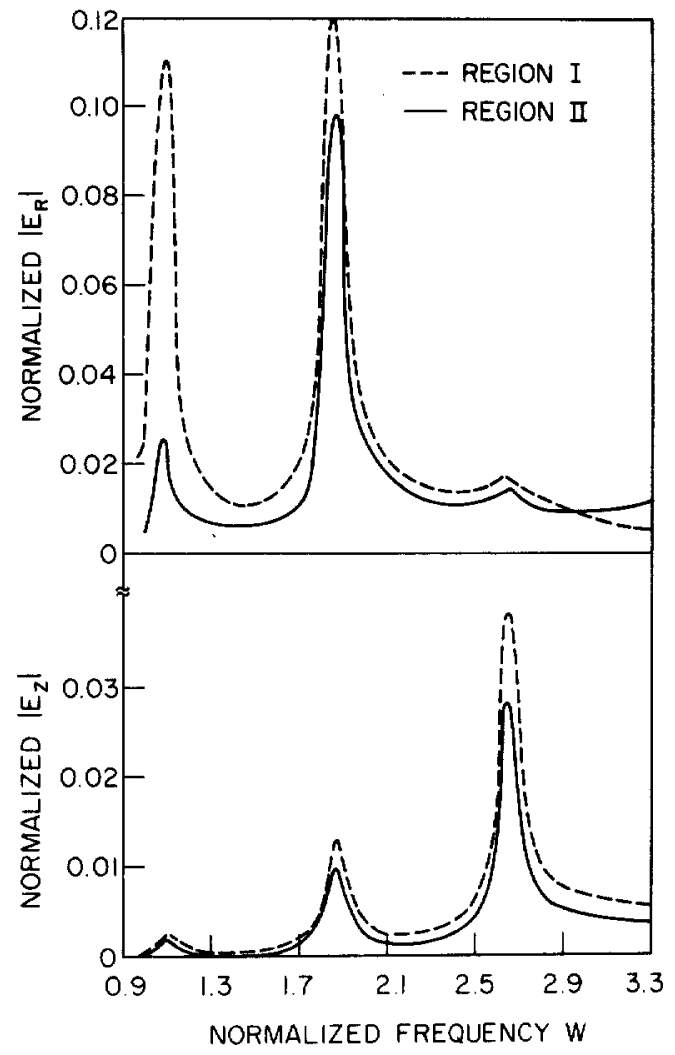

FIG. 7. Normalized amplitude of the radial and axial electric field at $r=0.5 b, r=2 b$, and $z=0.67 L$ as functions of frequency in an open beam-plasma waveguide. $\left(n_{p}=10^{9} \mathrm{~cm}^{-3}, b=3 \mathrm{~mm}\right.$, $\nu_{e N} / \omega_{p i}=0.1, I_{b}=2.5 \mathrm{~mA}, V_{b}=600 \mathrm{~V}, B_{0}=310 \mathrm{G}, \mathrm{H}^{+}$ion, and $L=61 \mathrm{~cm}$.)

large amplitude. The reason for this behavior may be explained by information from the theoretical curves given in Fig. 5 where the real parts of the radial and axial electric field are plotted as functions of the axial distance at the first three resonant frequencies. It is clear from Fig. 5 that the first, second, and third resonances are half-wave, full-wave, and three half-wave resonances, respectively. The axial electric field is maximum and the radial electric field is zero at the two conducting ends. It is noted that at the third resonant frequency the radial electric field has a node and the axial electric field has an antinode near the axial position of the observation point (coincident with the probe position in the experiment). The radial electric-field amplitude, therefore, is small at the third resonant frequency but the axial electric field has a relatively large amplitude at this frequency. Additional frequency response curves similar to Fig. 7 which show the effect of electron-neutral collisions have been given in Ref. 3 .

Examination of the frequency response curves of radial and axial electric fields given in Fig. 7 reveals that the resonant frequencies lie just above the ion plasma frequency. Since the resonant frequencies are half-wave, full-wave, and three half-wave resonances as shown in Fig. 5, it is concluded that these frequencies can be predicted quite accurately for any set of parameters from a dispersion diagram of the type shown in Fig. 6 by finding the frequencies where

or

$$
k_{z} L=n \pi, \quad n=1,2, \cdots,
$$

$$
L=n \lambda_{s} / 2,
$$

where the positive plasma wave root is used for $k_{z}$ and $L$ is the distance from the electron gun to the beam collector. The resonant frequencies found by this method lie very close to those obtained by the detailed normal-mode analysis. The effects of the variation of the plasma density, magnetic field, beam voltage, and ion mass on the first two resonant frequencies can, therefore, easily be predicted from a dispersion diagram by finding the resonant frequencies from the intersections of the lines $k_{z}=\pi / L$ and $k_{z}=2 \pi / L$ with the dispersion curves for the gases used experimentally (hydrogen, deuterium, neon, and argon). The resulting values of $k_{z}$ correspond to values of $K$ in the range of 1 to 4 in Fig. 6. Examples of this have previously been published. ${ }^{3,4}$ It is found that the resonant frequencies decrease with increasing ion mass but the amount of decrease is much less than the decrease in $\omega_{p i}$, and that the resonant frequencies decrease as the beam voltage is increased. For a given beam voltage, ion mass, and plasma dimensions, the resonant frequencies increase as the square root of the plasma density. The effect of the variation of the external dc magnetic field (or $\left.\omega_{c e} / \omega_{p e}\right)$ on the dispersion curve is negligible in the low-density regime. The resonant frequencies are, therefore, rather independent of the dc magnetic field.

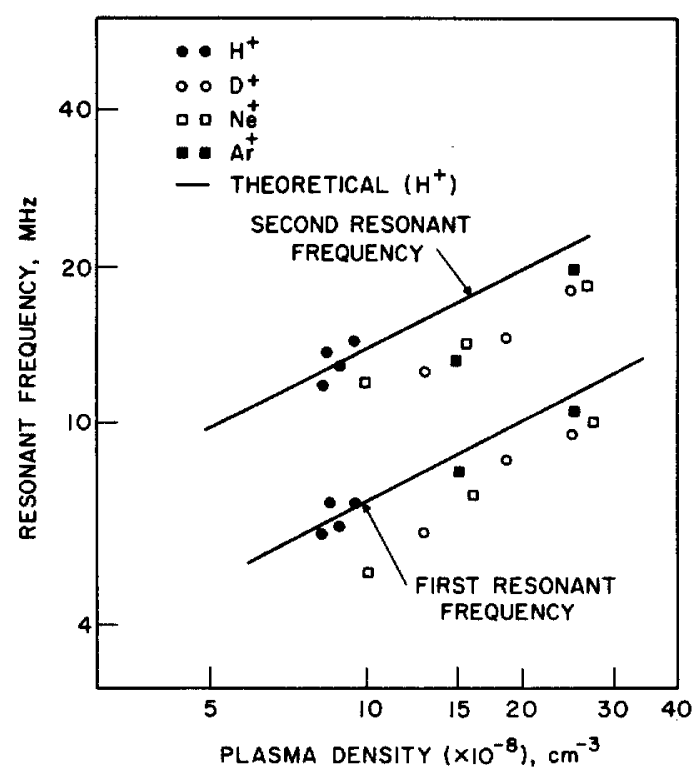

FIG. 8. Variation of resonant frequencies as a function of plasma density for hydrogen, deuterium, neon, and argon. 


\section{COMPARISON OF EXPERIMENTAL AND THEORETICAL RESULTS}

In this section the experimental results are compared with the predictions based on the normal-mode analysis given in Sec. III. Experimental and theoretical frequency-response curves, variations of if fields with radial and axial distances, and the effects of plasma density, ion mass, beam voltage, and magnetic field on the resonant frequencies are compared.

The theoretical radial of electric field outside the plasma (region II) is plotted in Fig. 7. The axial observation position in Fig. 7 coincides with that used for the Langmuir probe in the experiment. Typical probe response curves outside the plasma are given in Fig. 4. For example, the curve for $n_{p}=9.6 \times 10^{8} \mathrm{~cm}^{-3}$ shows resonances at 7.0,14.5, and 21.0 MHz. Radial if electric field frequency response curves calculated theoretically from the normal-mode field analysis for the experimental parameters accurately predict the resonant frequencies, but as shown in Fig. 7 the theoretical curve has sharper resonances than the experimental curve even when the effect of electron-neutral collisions is included. Electron-neutral collisions are dominant in the experiment and realistic values of collision frequency give $\nu_{e N} / \omega_{p i} \cong 0.25$, whereas the value used in computing the curves in Fig. 7 was $\nu_{e N} / \omega_{p i}=0.1$. Curves for ratios of 0.25 and 0.5 have previously been given. $^{3}$ The unnormalized field strengths at the first two resonances for the case of $\nu_{e N} / \omega_{p i}=0.25$ are 35 $\mathrm{V} / \mathrm{cm}$ and $48 \mathrm{~V} / \mathrm{cm}$, respectively. Estimation of the fields in this experiment and measurements in other experiments have indicated that the electric-field strength is of the same order of magnitude or even higher. $^{\text {? }}$

The axial distributions of the radial field at the first two resonances are compared with theory in Fig. 5. The quantity $\operatorname{Re}\left(E_{R}\right)$ plotted in the figure would be the output of a phase-sensitive detector and is in good agreement with the field strength measured by such means. The resonances occur when the length of the system is an integral number of half-wavelengths. The theoretically calculated and experimentally obtained radial variations of the field strength outside the plasma are of the same general shape, i.e., a decay of the field corresponding to that of the modified Bessel function $K_{1}$.

Figure 4 shows the rf field frequency response curves for a hydrogen plasma in which the parameter for the three curves is plasma density. By examination of this figure it is found that the resonant frequencies increase with increasing plasma density. Similar curves are obtained for deuterium, neon, and argon. The theoretical calculation predicts that the resonant frequencies should increase in proportion to the square root of the plasma density as shown by the straight line with a slope of $\frac{1}{2}$ drawn in Fig. 8 for the case of hydrogen

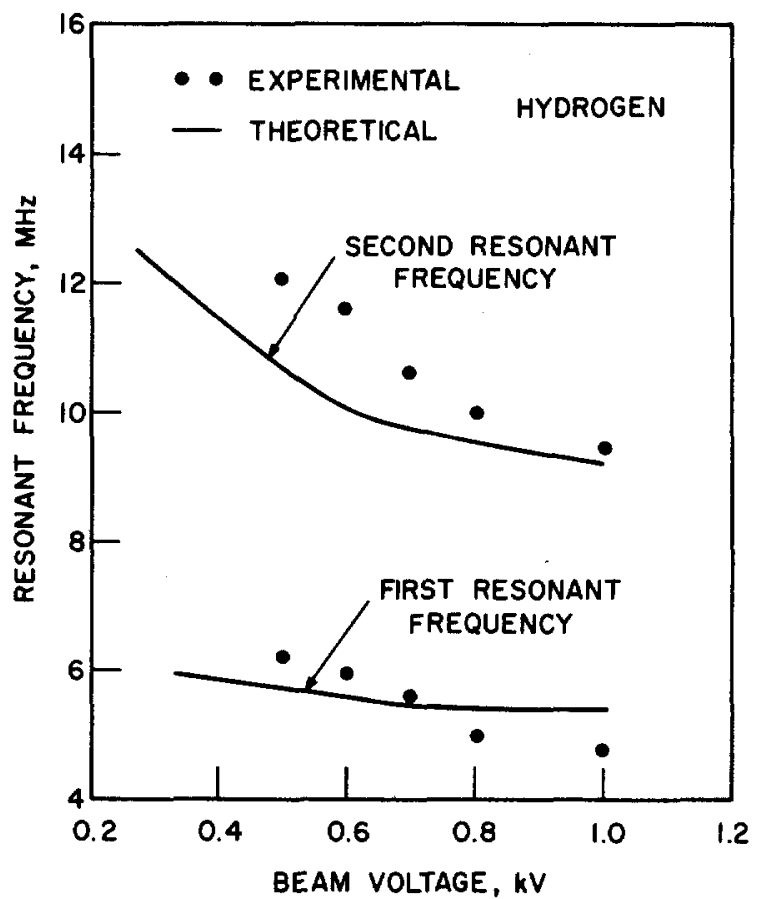

FiG. 9. Variation of resonant frequencies as a function of beam voltage for a hydrogen plasma.

ions. There is some scatter in the data but they confirm the theoretical prediction that the resonant frequencies increase in proportion to the square root of the plasma density. The results of the theoretical analysis predict that the resonant frequencies decrease with increasing ion mass but the decrease is much less than the decrease in the ion-plasma frequency. Such behavior is evident from the resonant frequency data plotted in Fig. 8. The experimental data show a decrease in the resonant frequency of the same order as that predicted; however, these factors are in the range of experimental error which occurs in the determination of plasma density. It is expected that calculations now in progress for nonuniform plasmas will result in theoretical lines in Fig. 8 which are slightly lower and closer to the data points.

The first and second resonant frequencies in the case of hydrogen plasma are plotted as functions of beam voltage in Fig. 9. The two curves show that the resonant frequencies decrease as the beam voltage is increased. The decrease in the second resonant frequency is more than that of the first for the same change of beam voltage. This behavior is predicted theoretically by finding the resonant frequencies from the dispersion diagram for various beam voltages. The continuous lines drawn in Fig. 9 represent the theoretical curves for the variation of the resonant frequencies as functions of beam voltage. The agreement between theory and experiment is good considering the influence of experimental errors in the measurement of plasma density. Experimentally, it was found that the resonant 
frequencies do not change appreciably with the change in the magnetic field for the range of parameters under study.

\section{v. DISCUSSION AND CONCLUSIONS}

The normal-mode analysis has been applied to other beam-plasma configurations, and the frequency range has been extended down through $\omega_{\mathrm{lb}}$ for both the low density range $\left(\omega_{\mathrm{l}} \cong \omega_{p i}\right)$ and the high-density range $\left[\omega_{\mathrm{lh}} \cong\left(\omega_{c e} \omega_{c i}\right)^{1 / 2}\right]$. The results show that at least for the cases studied a weaker "body" resonance does appear in the electric field at $\omega_{\mathrm{lh}}$ and, in addition, the geometric resonances are found. The dispersion equations have been given by Briggs ${ }^{14}$ and the details of the calculations may be found in Ref. 17 .

These analyses were carried out to determine if the electron beam will couple to the plasma at the lowerhybrid frequency in addition to exciting the geometric resonances. It has been suggested ${ }^{1-4}$ that an electron beam will act as an ac line-charge or current source and will excite the lower-hybrid resonance. The analysis of Ref. 17 shows that a resonance effect occurs at $\omega_{\mathrm{lh}}$ but apparently electron-beam excitation has not been observed experimentally. External electrode excitation has recently been reported. ${ }^{18}$ The electron-beam-excited geometric resonances have been found above $\omega_{p i}$ and in many cases at frequencies that are essentially independent of ion mass. Within the quasistatic approximation one may consider these geometric resonances to be at the lower-hybrid resonant frequency for oblique propagation as given by (1), which becomes independent of ion mass as $\theta$ changes from $90 \mathrm{deg}$. The plasma radius and length in the present experiment make $\theta$ small enough to be in this limit. These results suggest that a modulated electron beam may not efficiently couple to the body resonance at $\omega_{\mathrm{lh}}$, but that it does couple strongly to the geometric resonances. Auer et $a l .^{19}$ have pointed out that the effects of ac space charge and axial electric field are smaller in the highdensity.limit, and thus further experimental study with an electron beam should be worthwhile in a denser plasma to explore the frequency range around $\omega_{\mathrm{lh}}=$ $\left(\omega_{c o} \omega_{c i}\right)^{1 / 2}$. It should also be pointed out that the quasistatic assumption is in doubt in this frequency range, which is near a cutoff frequency of the plasma waveguide $\left(k_{z}=0\right)$, and that further theoretical studies should be made without this assumption.

The predictions based on the normal-mode analysis are in good agreement with the present experimental observations particularly with regard to the resonant frequencies, the relative of electric-field amplitudes, and their spatial variations. It is found that the inter- action encountered in the present beam-plasma system is that of the beam space-charge waves and not that of the cyclotron waves. The beam-plasma system is not absolutely unstable for the parameters of the present experiment. The gridded probe measurements show that energetic ions are produced by the modulated electron beam. Evidence of the production of energetic ions is also obtained by the observation of increased plasma diameter when the beam modulation is at one of the resonant frequencies. The theoretical analysis and experiment suggest that the ions are heated owing to the creation of a radial rf electric field (of the order of a few tens of $\mathrm{V} / \mathrm{cm}$ ) in the plasma. This radial electric field transfers energy to the ions. The excitation of $\mathrm{rf}$ fields near $\omega_{\mathrm{lb}}$ by a modulated electron beam within a plasma appears to have an advantage over those methods which utilize coils external to the plasma and thus must meet conditions of accessibility through the outer low-density region of the plasma. It has the disadvantage of introducing a beam into the plasma, but the beam need not be dense enough to excite strong beam-plasma instabilities.

\section{ACKNOWLEDGMENT}

This work was supported by the National Science Foundation under Research Grant No. GK-15689.

${ }^{1}$ G. M. Haas and M. Eisner, Phys. Fluids 14, 606 (1971).

${ }^{2}$ G. M. Haas and M. A. Eisner, Bull. Am. Phys. Soc. 13, 1517 (1968).

${ }^{3}$ V. P. Bhatnagar and W. D. Getty, Phys. Rev. Letters 26, 1527 (1971).

V. P. Bhatnagar and W. D. Getty, Phys. Fluids 15, 514 1972)

${ }_{5}^{5}$ G. M. Haas, W. B. Ard, M. C. Becker, R. A. Dandl, A. C. England, R. L. Livesey, O. D. Matlock, and M. W. McGuffin, Oak Ridge National Laboratory, Report No. ORNL 4063 (1967), p. 40 .

${ }^{6}$ G. M. Haas and R. A. Dandl, Phys. Fluids 10, 678 (1967).

7 D. R. Beeth, G. M. Haas, and M. Eisner, Bull. Am. Phys. Soc. 15, 1408 (1970).

${ }^{8}$ G. M. Haas, Phys. Fluids 12, 2455 (1969).

-W. P. Allis, S. J. Buchsbaum, and A. Bers, Waves in Anisotropic Plasmas (M.I.T. Press, Cambridge, Mass., 1963), Chap. 3.

${ }^{10}$ H. Groendijk, M. T. Vlaardingerbroek, and K. R. U. Weimer, Philips Res. Rep. 20, 485 (1965).

${ }^{11}$ D. A. Dunn, W. Nichparenko, J. E. Simpson, and K. I. Thomassen, J. Appl. Phys. 36, (1965).

${ }_{12}$ W. E. Scharfman, Phys. Fluids 11, 689 (190́8).

${ }^{13} \mathrm{~J}$. Frey and C. K. Birdsall, J. Appl. Phys. 37, 2051 (1966).

${ }_{14} \mathrm{R}$. J. Briggs, Electron-Stream Interaction with Plasmas (M.I.T. Press, Cambridge, Mass., 1964), Appendix G.

${ }^{15}$ S. A. Self, J. Appl. Phys. 40, 5217 (1969).

$16 \mathrm{~J}$. D. Gillanders (private communication).

${ }_{17}$ V. P. Bhatnagar, Ph.D. thesis, The University of Michigan, 1971.

${ }_{18}$ W. M. Hooke and S. Bernabei, Phys. Rev. Letters 28, 407 (1972).

${ }_{19}$ P. L. Auer, H. Hurwitz, Jr., and R. D. Miller, Phys. Fluids 1, 501 (1958). 